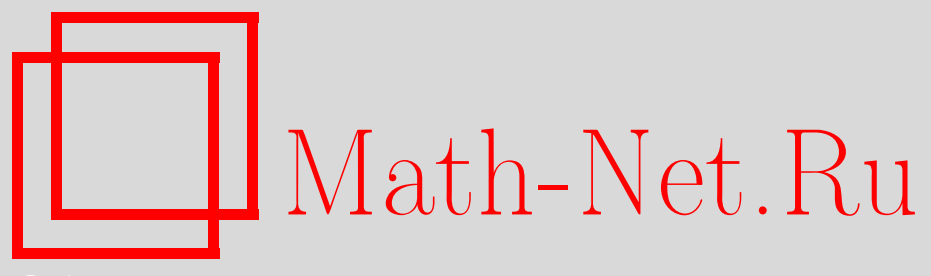

А. Лауринчикас, Совместная дискретная универсальность дзета-функций Гурвица, Матем. сб., 2014, том 205, номер 11, 75-94

DOI: https://doi.org/10.4213/sm8371

Использование Общероссийского математического портала Math-Net.Ru подразумевает, что вы прочитали и согласны с пользовательским соглашением http://www . mathnet.ru/rus/agreement

Параметры загрузки:

IP: 3.85 .5 .30

26 апреля 2023 г., 10:54:25

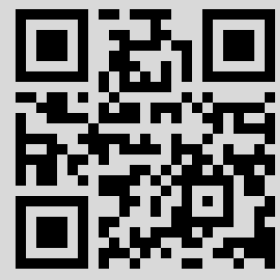




\section{А. Лауринчикас}

\section{Совместная дискретная универсальность дзета-функций Гурвица}

Получена совместная дискретная теорема универсальности для дзетафункций Гурвица. При этом параметры дзета-функций и шаг сдвигов этих функций, приближающих данный набор аналитических функций, связаны некоторым условием линейной независимости. Теорема Нестеренко дает пример, удовлетворяющий этому условию. Теорема универсальности применена для оценки числа нулей линейной комбинации дзетафункций Гурвица.

Библиография: 20 названий.

Ключевые слова: алгебраическая независимость, дзета-функция Гурвица, линейная независимость, предельная теорема, пространство аналитических функций, совместная универсальность.

DOI: $10.4213 / \mathrm{sm} 8371$

\section{§ 1. Введение}

Дзета-функция Гурвица $\zeta(s, \alpha), s=\sigma+i t$, с параметром $\alpha, 0<\alpha \leqslant 1$, при $\sigma>1$ определяется рядом Дирихле:

$$
\zeta(s, \alpha)=\sum_{m=0}^{\infty} \frac{1}{(m+\alpha)^{s}},
$$

и аналитически продолжается на всю комплексную плоскость, за исключением точки $s=1$, которая является простым полюсом с вычетом 1 . Имеем $\zeta(s, 1)=$ $\zeta(s)$ и

$$
\zeta\left(s, \frac{1}{2}\right)=\left(2^{s}-1\right) \zeta(s),
$$

где $\zeta(s)$ - дзета-функция Римана. Таким образом, дзета-функция Гурвица является обобщением дзета-функции Римана. Аналитические свойства функции $\zeta(s, \alpha)$ зависят от арифметики параметра $\alpha$.

В настоящей статье мы будем рассматривать свойство универсальности дзета-функций Гурвица, т.е. нас интересуют проблемы, связанные с приближением аналитических функций сдвигами $\zeta(s+i \tau, \alpha), \tau \in \mathbb{R}$, или $\zeta(s+i k h, \alpha)$, $k \in \mathbb{N}_{0}=\mathbb{N} \cup\{0\}$, где $h$ - фиксированное положительное число. В первом случае универсальность называется непрерывной, а во втором - дискретной. Наша цель - получить совместную дискретную теорему универсальности о приближении набора аналитических функций дискретными сдвигами дзета-функций Гурвица. Перед этим мы напомним некоторые известные результаты об универсальности функции $\zeta(s, \alpha)$. Пусть $D=\{s \in \mathbb{C}: 1 / 2<\sigma<1\}$. Через $\mathscr{K}$ будем обозначать класс компактных подмножеств полосы $D$, обладающих связным 
дополнением, а через $H(K), K \in \mathscr{K},-$ класс непрерывных в $K$ и аналитических внутри $K$ функций. Кроме того, пусть meas $A$ - мера Лебега измеримого множества $A \subset \mathbb{R}$. Непрерывная универсальность функции $\zeta(s, \alpha)$ описывается следующей теоремой.

ТЕОРема 1. Предположим, что $\alpha$ - трансцендентное или рациональное число, не равное $1,1 / 2$. Пусть $K \in \mathscr{K}$, a $f(s) \in H(K)$. Тогда для всякого $\varepsilon>0$

$$
\liminf _{T \rightarrow \infty} \frac{1}{T} \operatorname{meas}\left\{\tau \in[0, T]: \sup _{s \in K}|\zeta(s+i \tau, \alpha)-f(s)|<\varepsilon\right\}>0 .
$$

Неравенство теоремы показывает, что множество сдвигов, приближающих данную функцию $f(s) \in H(K)$, является бесконечным, оно имеет положительную нижнюю плотность.

Теорема 1 с рациональным $\alpha$ была получена независимо С. М. Ворониным в [1], С. М. Гонеком в [2] и Б. Багчи в [3]. Случай трансцендентного $\alpha$ содержится в [4]. Функция $\zeta(s, \alpha)$ также универсальна и при $\alpha=1,1 / 2$, но в этом случае приближаемая функция $f(s)$ не должна иметь нулей в $K$. Случай алгебраического иррационального $\alpha$ до сих пор является открытой проблемой.

Следующая теорема является дискретным вариантом теоремы 1.

Теорема 2. Предположим, что параметр $\alpha$, множество $K$ и функиия $f(s)$ те же самые, что и в теореме 1 . В случае рационального $\alpha$ пусть $h$ любое положительное число, а при трансцендентном $\alpha$ пусть $h>0$ такое, что $\exp \{2 \pi / h\}$ является рациональным числом. Тогда для всякого $\varepsilon>0$

$$
\liminf _{N \rightarrow \infty} \frac{1}{N+1} \#\left\{0 \leqslant k \leqslant N: \sup _{s \in K}|\zeta(s+i k h, \alpha)-f(s)|<\varepsilon\right\}>0 .
$$

Теорема 2 с рациональным $\alpha$ с незначительно отличающимися требованиями на множество $K$ была доказана в [3]. Другим методом этот случай был получен в [5]. С трансцендентным $\alpha$ теорема 2 является частным случаем дискретной теоремы универсальности из работы [6].

Совместная универсальность дзета-функций Гурвица изучалась в работах [7] и [8]. Пусть имеем набор дзета-функций Гурвица $\zeta\left(s, \alpha_{j}\right), j=1, \ldots, r$. Т. Накамура в [7] получил одновременное приближение набора аналитических функций класса $H(K), K \in \mathscr{K}$, сдвигами $\zeta\left(s+i \tau, \alpha_{j}\right), j=1, \ldots, r$, с алгебраически независимыми параметрами $\alpha_{1}, \ldots, \alpha_{r}$. В [8] изучался более общий случай. Пусть

$$
L\left(\alpha_{1}, \ldots, \alpha_{r}\right)=\left\{\log \left(m+\alpha_{j}\right): m \in \mathbb{N}_{0}, j=1, \ldots, r\right\} .
$$

Теорема 3 (см. [8]). Предположим, что множество $L\left(\alpha_{1}, \ldots, \alpha_{r}\right)$ линейно независимо над полем рациональных чисел $\mathbb{Q}$. Пусть $K_{j} \in \mathscr{K} u f_{j}(s) \in H\left(K_{j}\right)$, $j=1, \ldots, r$. Тогда для всякого $\varepsilon>0$

$$
\liminf _{T \rightarrow \infty} \frac{1}{T} \operatorname{meas}\left\{\tau \in[0, T]: \sup _{1 \leqslant j \leqslant r} \sup _{s \in K_{j}}\left|\zeta\left(s+i \tau, \alpha_{j}\right)-f_{j}(s)\right|<\varepsilon\right\}>0 .
$$

Через $H(D)$ будем обозначать пространство аналитических в $D$ функций, наделенное топологией равномерной сходимости на компактах. В статьях [9]-[11] были получены теоремы универсальности для функций $F(\zeta(s, \alpha))$ 
и $F_{r}\left(\zeta\left(s, \alpha_{1}\right), \ldots, \zeta\left(s, \alpha_{r}\right)\right)$, где $F: H(D) \rightarrow H(D)$ и $F_{r}: H^{r}(D) \rightarrow H(D)$ - некоторые операторы.

Отметим, что дискретные теоремы универсальности более удобны для практических применений. Например, в [12] дискретный вариант теоремы Воронина (см. [13]) об универсальности дзета-функции Римана был использован для оценки сложных интегралов по аналитическим кривым, встречаемых в квантовой механике.

Первый результат о совместной дискретной универсальности дзета-функций Гурвица был получен Й. Сандером и Й. Штойдингом в [5] с рациональным параметром $\alpha=a / q$. Пусть $\varphi(q)$ обозначает функцию Эйлера, $\chi_{j}$ пробегает $\varphi(q)$ различных характеров Дирихле по модулю $q$ и $\underline{M}$ - это $(\varphi(q) \times \varphi(q))$-матрица,

$$
\underline{M}=\left(\frac{\chi_{j}(a)}{\varphi(q)}\right)_{\substack{1 \leqslant j \leqslant \varphi(q) \\ 1 \leqslant a \leqslant q,(a, q)=1}} .
$$

Кроме того, при $f_{a}(s) \in H(K), K \in \mathscr{K}$, пусть

$$
\underline{f}=\left(q^{-s} f_{a}(s)\right)_{1 \leqslant a \leqslant q,(a, q)=1}^{\mathrm{T}} .
$$

Тогда имеет место следующее утверждение (см. [5]).

Теорема 4. Предположим, что $K \in \mathscr{K} u f_{a}(s) \in H(K)$ для всякого $а$, $1 \leqslant a \leqslant q,(a, q)=1$. Если все элементы матрици $\underline{M}^{-1} \underline{f}$ не обрашаются в нуль на множестве $K$, то для всякого $\varepsilon>0$

$$
\liminf _{N \rightarrow \infty} \frac{1}{N+1} \#\left\{1 \leqslant k \leqslant N: \max _{\substack{1 \leqslant a \leqslant q \\(a, q)=1}} \sup _{s \in K}\left|\zeta\left(s+i \Delta k, \frac{a}{q}\right)-f_{a}(s)\right|<\varepsilon\right\}>0,
$$

где $\Delta=2 \pi m / \log q$ с $m \in \mathbb{N}$, если $q \geqslant 2, u \Delta \neq 0$ в противном случае.

Сформулируем результат настоящей статьи - совместную дискретную теорему универсальности для дзета-функций Гурвица. Пусть $h>0$ и

$$
L\left(\alpha_{1}, \ldots, \alpha_{r} ; h\right)=\left\{\left(\log \left(m+\alpha_{j}\right): m \in \mathbb{N}_{0}, j=1, \ldots, r\right), \frac{\pi}{h}\right\} .
$$

ТЕОРема 5. Предположим, что множество $L\left(\alpha_{1}, \ldots, \alpha_{r} ; h\right)$ линейно независимо над $\mathbb{Q}$. Пусть $K_{j} \in \mathscr{K} u f_{j}(s) \in H\left(K_{j}\right), j=1, \ldots, r$. Тогда для всякого $\varepsilon>0$

$$
\liminf _{N \rightarrow \infty} \frac{1}{N+1} \#\left\{0 \leqslant k \leqslant N: \sup _{1 \leqslant j \leqslant r} \sup _{s \in K_{j}}\left|\zeta\left(s+i k h, \alpha_{j}\right)-f_{j}(s)\right|<\varepsilon\right\}>0 .
$$

В силу знаменитой теоремы Нестеренко (см. [14]) известно, что числа $\pi$, $e^{\pi}$ и $\Gamma(1 / 4)$ алгебраически независимы над $\mathbb{Q}$. Поэтому при $\alpha_{1}=\pi / 4, \alpha_{2}=$ $(1 / 6) \Gamma(1 / 4)$ и рациональном $h>0$ множество $L\left(\alpha_{1}, \alpha_{2} ; h\right)$ линейно независимо над $\mathbb{Q}$ и для дзета-функций $\zeta\left(s, \alpha_{1}\right)$ и $\zeta\left(s, \alpha_{2}\right)$ имеет место утверждение теоремы 5 .

Приведем один пример применения теоремы 5 . Пусть $\underline{\alpha}=\left(\alpha_{1}, \ldots, \alpha_{r}\right)$ и

$$
\widehat{\zeta}(s, \underline{\alpha})=c_{1} \zeta\left(s, \alpha_{1}\right)+\cdots+c_{r} \zeta\left(s, \alpha_{r}\right)
$$

с $c_{j} \in \mathbb{C}$, причем $c_{j} \neq 0$ хотя бы при одном $j=1, \ldots, r, \underline{c}=\left(c_{1}, \ldots, c_{r}\right)$. 
Теорема 6. Предположим, что множество $L\left(\alpha_{1}, \ldots, \alpha_{r} ; h\right)$ линейно независимо над $\mathbb{Q}$. Тогда для всех $\sigma_{1}, \sigma_{2}, 1 / 2<\sigma_{1}<\sigma_{2}<1$, существует констанma $c=c\left(\sigma_{1}, \sigma_{2}, \underline{\alpha}, \underline{c}\right)>0$ такая, что при достаточно большом $N \in \mathbb{N}$ функиия $\widehat{\zeta}(s+i k h, \underline{\alpha})$ имеет нуль в круге

$$
|s-\widehat{\sigma}| \leqslant \frac{\sigma_{2}-\sigma_{1}}{2}, \quad \widehat{\sigma}=\frac{\sigma_{1}+\sigma_{2}}{2},
$$

для более чем $c N$ чисел $k, 0 \leqslant k \leqslant N$.

\section{§ 2. Основная лемма}

Доказательство теоремы 5 основано на многомерной предельной теореме о слабой сходимости вероятностных мер в пространстве $H^{r}(D)$. В настоящей статье мы докажем предельную теорему на $r$-мерном произведении бесконечномерных торов. При этом существенно будем использовать линейную независимость множества $L\left(\alpha_{1}, \ldots, \alpha_{r} ; h\right)$. Через $\mathscr{B}(X)$ будем обозначать $\sigma$-поле борелевских множеств пространства $X$. Пусть $\gamma=\{s \in \mathbb{C}:|s|=1\}$ - единичная окружность на комплексной плоскости и

$$
\Omega=\prod_{m=0}^{\infty} \gamma_{m},
$$

где $\gamma_{m}=\gamma$ для всех $m \in \mathbb{N}_{0}$. В силу известной теоремы Тихонова бесконечномерный тор $\Omega$ с топологией произведения и операцией поточечного перемножения является компактной топологической абелевой группой. Поэтому на $(\Omega, \mathscr{B}(\Omega))$ может быть определена вероятностная мера Хаара. Пусть

$$
\underline{\Omega}=\Omega_{1} \times \cdots \times \Omega_{r},
$$

где $\Omega_{j}=\Omega$ при $j=1, \ldots, r$. Тогда опять из теоремы Тихонова вытекает, что $\underline{\Omega}$ - компактная топологическая абелева группа. Пусть $m_{H}$ - вероятностная мера Хаара на $(\underline{\Omega}, \mathscr{B}(\underline{\Omega}))$. Получаем вероятностное пространство $\left(\underline{\Omega}, \mathscr{B}(\underline{\Omega}), m_{H}\right)$. Замечаем, что мера $m_{H}$ является произведением мер Хаара на $\left(\Omega_{j}, \mathscr{B}\left(\Omega_{j}\right)\right)$, $j=1, \ldots, r$.

Через $\omega_{j}(m)$ обозначим проекцию элемента $\omega_{j} \in \Omega_{j}$ на координатное пространство $\gamma_{m}, m \in \mathbb{N}_{0}, j=1, \ldots, r$. Элементы $\underline{\omega}$ тора $\underline{\Omega}$ имеют вид $\underline{\omega}=$ $\left(\omega_{1}, \ldots, \omega_{r}\right)$, где $\omega_{j} \in \Omega_{j}, j=1, \ldots, r$. Докажем слабую сходимость меры

$$
\begin{gathered}
Q_{N}(A)=\frac{1}{N+1} \#\left\{0 \leqslant k \leqslant N:\left(\left(\left(m+\alpha_{1}\right)^{-i k h}: m \in \mathbb{N}_{0}\right), \ldots,\right.\right. \\
\left.\left.\left(\left(m+\alpha_{r}\right)^{-i k h}: m \in \mathbb{N}_{0}\right)\right) \in A\right\}, \quad A \in \mathscr{B}(\underline{\Omega}) .
\end{gathered}
$$

Лемма 1. Предположим, что множество $L\left(\alpha_{1}, \ldots, \alpha_{r} ; h\right)$ линейно независимо над $\mathbb{Q}$. Тогда $Q_{N}$ при $N \rightarrow \infty$ слабо сходится $к$ мере Хаара $m_{H}$.

ДокАзАтЕльство. Дуальная группа группы $\underline{\Omega}$ изоморфна группе

$$
\mathscr{D}=\bigoplus_{j=1}^{r}\left(\bigoplus_{m \in \mathbb{N}_{0}} \mathbb{Z}_{j}\right),
$$


где $\mathbb{Z}_{j}=\mathbb{Z}, j=1, \ldots, r$. Элемент $\underline{k}=\left(\underline{k}_{1}, \ldots, \underline{k}_{j}\right) \in \mathscr{D}, \underline{k}_{j}=\left(k_{j m}: m \in \mathbb{N}_{0}\right)$, $j=1, \ldots, r$, действует на $\underline{\Omega}$ по правилу

$$
\underline{k} \rightarrow \underline{\omega}^{\underline{k}}=\prod_{j=1}^{r} \prod_{m=0}^{\infty} \omega_{j}^{k_{j m}}(m),
$$

где только конечное число целых чисел $k_{j m}$ отличны от нуля. Следовательно, преобразование Фурье $g_{N}(\underline{k})$ меры $Q_{N}$ имеет вид

$$
g_{N}(\underline{k})=\int_{\underline{\Omega}} \prod_{j=1}^{r} \prod_{m=0}^{\infty} \omega_{j}^{k_{j m}}(m) \mathrm{d} Q_{N} .
$$

Отсюда и из определения $Q_{N}$ находим

$$
\begin{aligned}
g_{N}(\underline{k}) & =\frac{1}{N+1} \sum_{k=0}^{N} \prod_{j=1}^{r} \prod_{m=0}^{\infty}\left(m+\alpha_{j}\right)^{-i h k k_{j m}} \\
& =\frac{1}{N+1} \sum_{k=0}^{N} \exp \left\{-i k h \sum_{j=1}^{r} \sum_{m=0}^{\infty} k_{j m} \log \left(m+\alpha_{j}\right)\right\},
\end{aligned}
$$

где только конечное число целых чисел $k_{j m}$ отличны от нуля. Из линейной независимости множества $L\left(\alpha_{1}, \ldots, \alpha_{r} ; h\right)$ следует линейная независимость множества

$$
L\left(\alpha_{1}, \ldots, \alpha_{r}\right)=\left\{\log \left(m+\alpha_{j}\right): m \in \mathbb{N}_{0}, j=1, \ldots, r\right\} .
$$

Поэтому

$$
\sum_{j=1}^{r} \sum_{m=0}^{\infty} k_{j m} \log \left(m+\alpha_{j}\right)=0
$$

тогда и только тогда, когда $\underline{k}=\underline{0}$. Замечаем, что в условиях леммы при $\underline{k} \neq \underline{0}$

$$
\exp \left\{-i h \sum_{j=1}^{r} \sum_{m=0}^{\infty} k_{j m} \log \left(m+\alpha_{j}\right)\right\} \neq 1 .
$$

Действительно, если неравенство (2.3) не выполняется, то существует целое $l \neq 0$ такое, что

$$
\exp \left\{\sum_{j=1}^{r} \sum_{m=0}^{\infty} k_{j m} \log \left(m+\alpha_{j}\right)\right\}=\exp \left\{\frac{2 \pi l}{h}\right\} .
$$

Отсюда

$$
\sum_{j=1}^{r} \sum_{m=0}^{\infty} k_{j m} \log \left(m+\alpha_{j}\right)-l \frac{2 \pi}{h}=0,
$$

что противоречит линейной независимости над $\mathbb{Q}$ множества $L\left(\alpha_{1}, \ldots, \alpha_{r} ; h\right)$. Из (2.1), (2.3) и (2.2) получаем

$$
g_{N}(\underline{k})= \begin{cases}\frac{1-(N+1) i h \sum_{j=1}^{r} \sum_{m=0}^{\infty} k_{j m} \log \left(m+\alpha_{j}\right)}{(N+1)\left(1-i h \sum_{j=1}^{r} \sum_{m=0}^{\infty} k_{j m} \log \left(m+\alpha_{j}\right)\right)}, & \text { если } \underline{k}=\underline{0}, \\ 1, & \text { если } \underline{k} \neq \underline{0} .\end{cases}
$$


Таким образом, имеем

$$
\lim _{N \rightarrow \infty} g_{N}(\underline{k})= \begin{cases}1, & \text { если } \underline{k}=\underline{0}, \\ 0, & \text { если } \underline{k} \neq \underline{0} .\end{cases}
$$

Отсюда и из теоремы непрерывности для вероятностных мер на компактных группах (см., например, [15; теорема 1.4.2]) получаем утверждение леммы.

\section{§ 3. Предельная теорема}

На вероятностном пространстве $\left(\underline{\Omega}, \mathscr{B}(\underline{\Omega}), m_{H}\right)$ определим $H^{r}(D)$-значный случайный элемент $\underline{\zeta}(s, \underline{\alpha}, \underline{\omega})$ формулой

$$
\underline{\zeta}(s, \underline{\alpha}, \underline{\omega})=\left(\zeta\left(s, \alpha_{1}, \omega_{1}\right), \ldots, \zeta\left(s, \alpha_{r}, \omega_{r}\right)\right),
$$

где

$$
\zeta\left(s, \alpha_{j}, \omega_{j}\right)=\sum_{m=0}^{\infty} \frac{\omega_{j}(m)}{\left(m+\alpha_{j}\right)^{s}}, \quad j=1, \ldots, r .
$$

Ряды, определяющие $\zeta\left(s, \alpha_{j}, \omega_{j}\right)$, для почти всех $\omega_{j} \in \Omega_{j}$ сходятся равномерно на компактных подмножествах полосы $D$ и определяют $H(D)$-значные случайные элементы на $\left(\Omega_{j}, \mathscr{B}\left(\Omega_{j}\right), m_{j H}\right)$, где $m_{j H}$ - мера Хаара на $\left(\Omega_{j}, \mathscr{B}\left(\Omega_{j}\right)\right)$, $j=1, \ldots, r$ (см. [4]). Через $P_{\underline{\zeta}}$ обозначим распределение случайного элемента $\underline{\zeta}(s, \underline{\alpha}, \underline{\omega})$, т.е.

$$
P_{\underline{\zeta}}(A)=m_{H}(\underline{\omega} \in \underline{\Omega}: \underline{\zeta}(s, \underline{\alpha}, \underline{\omega}) \in A), \quad A \in \mathscr{B}\left(H^{r}(D)\right) .
$$

Докажем слабую сходимость вероятностной меры

$$
P_{N}(A) \stackrel{\text { def }}{=} \frac{1}{N+1} \#\{0 \leqslant k \leqslant N: \underline{\zeta}(s+i k h, \underline{\alpha}) \in A\}, \quad A \in \mathscr{B}\left(H^{r}(D)\right),
$$

где

$$
\underline{\zeta}(s, \underline{\alpha})=\left(\zeta\left(s, \alpha_{1}\right), \ldots, \zeta\left(s, \alpha_{r}\right)\right) .
$$

Теорема 7. Предположим, что множество $L\left(\alpha_{1}, \ldots, \alpha_{r} ; h\right)$ линейно независимо над $\mathbb{Q}$. Тогда $P_{N}$ при $N \rightarrow \infty$ слабо сходится $к P_{\underline{\zeta}}$.

K сожалению, мы не знаем прямого метода доказательства теоремы 7 , основанного на сходимости некоторых преобразований. Поэтому мы будем пользоваться сохранением слабой сходимости при непрерывных отображениях, а также сходимостью по распределению для близких в некотором смысле случайных элементов.

Начнем с применения леммы 1 для функций, заданных абсолютно сходящимися рядами Дирихле. Берем фиксированное число $\sigma_{0}>1 / 2$ и полагаем

$$
v_{n}\left(m, \alpha_{j}\right)=\exp \left\{-\left(\frac{m+\alpha_{j}}{n+\alpha_{j}}\right)^{\sigma_{0}}\right\}, \quad m \in \mathbb{N}_{0}, \quad n \in \mathbb{N} .
$$

Пусть

$$
\begin{gathered}
\underline{\zeta}_{n}(s, \underline{\alpha})=\left(\zeta_{n}\left(s, \alpha_{1}\right), \ldots, \zeta_{n}\left(s, \alpha_{r}\right)\right), \\
\underline{\zeta}_{n}(s, \underline{\alpha}, \underline{\omega})=\left(\zeta_{n}\left(s, \alpha_{1}, \omega_{1}\right), \ldots, \zeta_{n}\left(s, \alpha_{r}, \omega_{r}\right)\right),
\end{gathered}
$$


где

$$
\zeta_{n}\left(s, \alpha_{j}\right)=\sum_{m=0}^{\infty} \frac{v_{n}\left(m, \alpha_{j}\right)}{\left(m+\alpha_{j}\right)^{s}}, \quad \zeta_{n}\left(s, \alpha_{j}, \omega_{j}\right)=\sum_{m=0}^{\infty} \frac{\omega_{j}(m) v_{n}\left(m, \alpha_{j}\right)}{\left(m+\alpha_{j}\right)^{s}},
$$

$j=1, \ldots, r$. Тогда имеем, что ряды, определяющие $\zeta_{n}\left(s, \alpha_{j}\right)$ и $\zeta_{n}\left(s, \alpha_{j}, \omega_{j}\right)$, $j=1, \ldots, r$, абсолютно сходятся в полуплоскости $\sigma>1 / 2$ (см. [4]).

Пусть $\underline{\widehat{\omega}}=\left(\widehat{\omega}_{1}, \ldots, \widehat{\omega}_{r}\right)-$ фиксированный элемент группы $\underline{\Omega}$. На $\left(H^{r}(D)\right.$, $\left.\mathscr{B}\left(H^{r}(D)\right)\right)$ определим две меры

$$
\begin{gathered}
P_{N, n}(A)=\frac{1}{N+1} \#\left\{0 \leqslant k \leqslant N: \underline{\zeta}_{n}(s+i k h, \alpha) \in A\right\}, \\
\widehat{P}_{N, n}(A)=\frac{1}{N+1} \#\left\{0 \leqslant k \leqslant N: \underline{\zeta}_{n}(s+i k h, \alpha, \widehat{\omega}) \in A\right\} .
\end{gathered}
$$

Лемма 2. Предположим, что множество $L\left(\alpha_{1}, \ldots, \alpha_{r} ; h\right)$ линейно независимо над $\mathbb{Q}$. Тогда на $\left(H^{r}(D), \mathscr{B}\left(H^{r}(D)\right)\right)$ существует вероятностная мера $P_{n}$ такая, что мерь $P_{N, n}$ и $\widehat{P}_{N, n}$ при $N \rightarrow \infty$ слабо сходятся $\kappa P_{n}$.

ДокАЗАтЕльство. Определим отображение $h_{n}: \underline{\Omega} \rightarrow H^{r}(D)$ формулой

$$
h_{n}(\omega)=\underline{\zeta}_{n}(s, \underline{\alpha}, \underline{\omega}) .
$$

Поскольку ряды, определяющие $\zeta\left(s, \alpha_{j}, \omega_{j}\right), j=1, \ldots, r$, сходятся абсолютно при $\sigma>1 / 2$, то из определения топологии произведения вытекает непрерывность отображения $h_{n}$. Кроме того, имеем

$$
h_{n}\left(\left(\left(m+\alpha_{1}\right)^{-i k h}: m \in \mathbb{N}_{0}\right), \ldots,\left(\left(m+\alpha_{r}\right)^{-i k h}: m \in \mathbb{N}_{0}\right)\right)=\underline{\zeta}_{n}(s+i k h, \underline{\alpha}) .
$$

Поэтому в обозначениях леммы 1 для всякого $A \in \mathscr{B}\left(H^{r}(D)\right)$

$$
\begin{aligned}
P_{N, n}(A)= & \frac{1}{N+1} \#\left\{0 \leqslant k \leqslant N:\left(\left(\left(m+\alpha_{1}\right)^{-i k h}: m \in \mathbb{N}_{0}\right), \ldots,\right.\right. \\
& \left.\left.\left(\left(m+\alpha_{r}\right)^{-i k h}: m \in \mathbb{N}_{0}\right)\right) \in h_{n}^{-1} A\right\} \\
= & Q_{N}\left(h_{n}^{-1} A\right)=Q_{N} h_{n}^{-1}(A) .
\end{aligned}
$$

Отсюда, из непрерывности отображения $h_{n}$, леммы 1 и теоремы 5.1 из [16] вытекает, что мера $P_{N, n}$ при $N \rightarrow \infty$ слабо сходится к мере $m_{H} h_{n}^{-1}$.

Осталось показать, что мера $\widehat{P}_{N, n}$ при $N \rightarrow \infty$ также слабо сходится к $m_{H} h_{n}^{-1}$. Определим отображение $h_{n}: \underline{\Omega} \rightarrow H^{r}(D)$ формулой

$$
\widehat{h}_{n}(\underline{\omega})=\underline{\zeta}_{n}(s, \underline{\alpha}, \underline{\widehat{\omega} \omega}) \text {. }
$$

Тогда $\widehat{h}_{n}$ непрерывно и

$$
\widehat{h}_{n}\left(\left(\left(m+\alpha_{1}\right)^{-i k h}: m \in \mathbb{N}_{0}\right), \ldots,\left(\left(m+\alpha_{r}\right)^{-i k h}: m \in \mathbb{N}_{0}\right)\right)=\underline{\zeta}_{n}(s+i k h, \underline{\alpha}, \underline{\widehat{\omega}}) .
$$

Следовательно, для всякого $A \in \mathscr{B}\left(H^{r}(D)\right)$

$$
\widehat{P}_{N, n}(A)=Q_{N} \widehat{h}_{n}^{-1}(A) .
$$

Отсюда аналогично случаю меры $P_{N, n}$ находим, что $\widehat{P}_{N, n}$ при $N \rightarrow \infty$ слабо сходится к мере $m_{H} \widehat{h}_{n}^{-1}$. Пусть отображение $u: \underline{\Omega} \rightarrow \underline{\Omega}$ задано формулой $u(\underline{\omega})=\underline{\omega} \underline{\widehat{\omega}}$. Тогда имеем $\widehat{h}_{n}(\underline{\omega})=h_{n}(u(\underline{\omega}))$. Хорошо известно, что мера Хаара $m_{H}$ является инвариантной относительно сдвигов точками из $\Omega$. Поэтому

$$
m_{H} \widehat{h}_{n}^{-1}=m_{H}\left(h_{n} u\right)^{-1}=\left(m_{H} u^{-1}\right) h_{n}^{-1}=m_{H} h_{n}^{-1} .
$$

Таким образом, обе меры $P_{N, n}$ и $\widehat{P}_{N, n}$ при $N \rightarrow \infty$ слабо сходятся к той же самой мере $P_{n}=m_{H} h_{n}^{-1}$. 
Переход от функции $\underline{\zeta}_{n}(s, \underline{\alpha})$ к функции $\underline{\zeta}(s, \underline{\alpha})$ требует некоторой оценки близости между $\underline{\zeta}(s, \underline{\alpha})$ и $\underline{\zeta}_{n}(s, \underline{\alpha})$. Для этого нам нужна метрика пространства $H^{r}(D)$. Известно, что существует последовательность компактных множеств $\left\{K_{l}: l \in \mathbb{N}\right\} \subset D$ такая, что

$$
D=\bigcup_{l=1}^{\infty} K_{l}
$$

$K_{l} \subset K_{l+1}$ для всех $l \in \mathbb{N}$, и если $K-$ компактное множество полосы $D$, то $K \subset K_{l}$ при некотором $l \in \mathbb{N}$. Пусть для $g_{1}, g_{2} \in H(D)$

$$
\rho\left(g_{1}, g_{2}\right)=\sum_{l=1}^{\infty} 2^{-l} \frac{\sup _{s \in K_{l}}\left|g_{1}(s)-g_{2}(s)\right|}{1+\sup _{s \in K_{l}}\left|g_{1}(s)-g_{2}(s)\right|} .
$$

Тогда $\rho$ является метрикой в пространстве $H(D)$, индуцирующей его топологию равномерной сходимости на компактах. Полагая

$$
\underline{\rho}\left(\underline{g}_{1}, \underline{g}_{2}\right)=\max _{1 \leqslant j \leqslant r} \rho\left(g_{1 j}, g_{2 j}\right),
$$

где $\underline{g}_{1}=\left(g_{11}, \ldots, g_{1 r}\right), \underline{g}_{2}=\left(g_{21}, \ldots, g_{2 r}\right), g_{1}, g_{2} \in H^{r}(D)$, имеем метрику в пространстве $H^{r}(D)$, индуцирующую его топологию.

Нам будет полезной лемма Галлахера, связывающая среднее квадратическое некоторых функций с его дискретным вариантом.

Лемма 3. Пусть $T_{0}, T \geqslant \delta>0$ - вещественные числа, $A$ - какое-либо конечное множество чисел отрезка $\left[T_{0}+\delta / 2, T_{0}+T-\delta / 2\right], \eta>0 u$

$$
N_{\delta}(x)=\sum_{\substack{t \in A \\|t-x|<\delta}} 1
$$

Пусть $S$ - комплекснозначная функиия, непрерывная на отрезке $\left[T_{0}, T_{0}+T\right]$ и обладающая непрерьвной производной в интервале $\left(T_{0}, T_{0}+T\right)$. Тогда

$$
\begin{aligned}
\sum_{t \in A} N_{\delta}^{-1}(t)|S(t)|^{2} \leqslant & \frac{1}{\delta} \int_{T_{0}}^{T_{0}+T}|S(x)|^{2} \mathrm{~d} x \\
& +\left(\int_{T_{0}}^{T_{0}+T}|S(x)|^{2} \mathrm{~d} x \int_{T_{0}}^{T_{0}+T}\left|S^{\prime}(x)\right|^{2} \mathrm{~d} x\right)^{1 / 2}
\end{aligned}
$$

Доказательство леммы содержится в [17; лемма 1.4].

Имеет место следующее приближение в среднем функции $\zeta(s, \underline{\alpha})$ функцией $\zeta_{n}(s, \underline{\alpha})$.

Лемма 4. При любъх $h>0 u \alpha_{j}, 0<\alpha_{j}<1, j=1, \ldots, r$, имеет место равенство

$$
\lim _{n \rightarrow \infty} \limsup _{N \rightarrow \infty} \frac{1}{N+1} \sum_{k=0}^{\infty} \underline{\rho}\left(\underline{\zeta}(s+i k h, \underline{\alpha}), \underline{\zeta}_{n}(s+i k h, \underline{\alpha})\right)=0 .
$$

ДокАзАТЕЛЬСтво. Из определения метрики $\underline{\rho}$ следует, что

$$
\begin{aligned}
& \frac{1}{N+1} \sum_{k=0}^{N} \underline{\rho}\left(\underline{\zeta}(s+i k h, \underline{\alpha}), \underline{\zeta}_{n}(s+i k h, \underline{\alpha})\right) \\
& \quad \leqslant \sum_{j=1}^{r} \frac{1}{N+1} \sum_{k=0}^{N} \rho\left(\zeta\left(s+i k h, \alpha_{j}\right), \zeta_{n}\left(s+i k h, \alpha_{j}\right)\right) .
\end{aligned}
$$


Поэтому достаточно показать, что при любых $h>0$ и $0<\alpha<1$

$$
\lim _{n \rightarrow \infty} \limsup _{N \rightarrow \infty} \frac{1}{N+1} \sum_{k=0}^{N} \rho\left(\zeta(s+i k h, \alpha), \zeta_{n}(s+i k h, \alpha)\right)=0 .
$$

Пусть $\sigma, 1 / 2<\sigma<1,-$ фиксированное число. Хорошо известно, что

$$
\int_{0}^{T}|\zeta(\sigma+i t, \alpha)|^{2} \mathrm{~d} t=O(T)
$$

Отсюда, применяя интегральную формулу Коши, находим оценку

$$
\int_{0}^{T}\left|\zeta^{\prime}(\sigma+i t, \alpha)\right|^{2} \mathrm{~d} t=O(T)
$$

Оценки (3.2), (3.3) и лемма 3 приводят к оценке

$$
\begin{aligned}
\sum_{k=0}^{N} \mid \zeta(\sigma & +i k h+i t, \alpha)\left.\right|^{2} \leqslant \frac{1}{h} \int_{0}^{N h}|\zeta(\sigma+i \tau+i t, \alpha)|^{2} \mathrm{~d} \tau \\
& +\left(\int_{0}^{N h}|\zeta(\sigma+i \tau+i t, \alpha)|^{2} \mathrm{~d} \tau \int_{0}^{N h}\left|\zeta^{\prime}(\sigma+i \tau+i t, \alpha)\right|^{2} \mathrm{~d} \tau\right)^{1 / 2} \\
= & O(N(1+|t|)) .
\end{aligned}
$$

Пусть $\sigma_{0}$ - число из определения $v_{n}(m, \alpha)$ и

$$
l_{n}(s, \alpha)=\frac{s}{\sigma_{0}} \Gamma\left(\frac{s}{\sigma_{0}}\right)(n+\alpha)^{s}, \quad n \in \mathbb{N},
$$

где $\Gamma(s)$ обозначает гамма-функцию Эйлера. Тогда применение формулы Меллина

$$
\frac{1}{2 \pi i} \int_{c-i \infty}^{c+i \infty} \Gamma(s) a^{-s} \mathrm{~d} s=\mathrm{e}^{-a}, \quad a, c>0,
$$

дает интегральное представление для $\zeta_{n}(s, \alpha)$

$$
\zeta_{n}(s, \alpha)=\frac{1}{2 \pi i} \int_{\sigma_{0}-i \infty}^{\sigma_{0}+i \infty} \zeta(s+z, \alpha) l_{n}(z, \alpha) \frac{\mathrm{d} z}{z} .
$$

Пусть $1 / 2<\sigma_{1}<1$ такое, что $\sigma_{1}<\sigma$. Тогда, передвигая прямую интегрирования в (3.5) налево, получаем

$$
\zeta_{n}(s, \alpha)-\zeta(s, \alpha)=\int_{\sigma_{1}-\sigma-i \infty}^{\sigma_{1}-\sigma+i \infty} \zeta(s+z, \alpha) l_{n}(z, \alpha) \frac{\mathrm{d} z}{z}+R_{n}(s, \alpha),
$$

где

$$
R_{n}(s, \alpha)=\operatorname{Re} s_{z=1-s} \zeta(s+z, \alpha) l_{n}(z, \alpha) z^{-1} .
$$

Берем любое компактное множество $K$ полосы $D$. Пусть $L \subset D$ - замкнутый ограниченный контур, окаймляющий множество $K$, а $\delta$-расстояние от $L$ до $K$. Тогда в силу интегральной формулы Коши

$$
\sup _{s \in K}\left|\zeta(s+i k h, \alpha)-\zeta_{n}(s+i k h, \alpha)\right| \leqslant \frac{1}{2 \pi \delta} \int_{L}\left|\zeta(z+i k h, \alpha)-\zeta_{n}(z+i k h, \alpha)\right||\mathrm{d} z| .
$$


Следовательно,

$$
\begin{aligned}
& \frac{1}{N+1} \sum_{k=0}^{N} \sup _{s \in K}\left|\zeta(s+i k h, \alpha)-\zeta_{n}(s+i k h, \alpha)\right| \\
& \quad=O\left(\frac{1}{N} \int_{L}|\mathrm{~d} z| \sum_{k=0}^{N}\left|\zeta(z+i k h, \alpha)-\zeta_{n}(z+i k h, \alpha)\right|\right) \\
& \quad=O\left(\frac{1}{N} \sup _{s \in L} \sum_{k=0}^{N}\left|\zeta(\sigma+i t+i k h, \alpha)-\zeta_{n}(\sigma+i t+i k h, \alpha)\right|\right) .
\end{aligned}
$$

Из равенства (3.6) следует

$$
\begin{aligned}
& \zeta(\sigma+i t+i k h, \alpha)-\zeta_{n}(\sigma+i t+i k h, \alpha)=-R_{n}(\sigma+i t+i k h, \alpha) \\
& +O\left(\int_{-\infty}^{\infty}\left|\zeta\left(\sigma_{1}+i t+i k h+i \tau, \alpha\right)\right|\left|l_{n}\left(\sigma_{1}-\sigma+i \tau, \alpha\right)\right| \mathrm{d} \tau\right) .
\end{aligned}
$$

Отсюда получаем

$$
\begin{aligned}
\frac{1}{N} \sum_{k=0}^{N}\left|\zeta(\sigma+i t+i k h, \alpha)-\zeta_{n}(\sigma+i t+i k h, \alpha)\right| \\
=O\left(\frac{1}{N} \sum_{k=0}^{N}\left|R_{n}(\sigma+i t+i k h, \alpha)\right|\right) \\
\quad+O\left(\int_{-\infty}^{\infty}\left|l_{n}\left(\sigma_{1}-\sigma+i \tau, \alpha\right)\right|\left(\frac{1}{N} \sum_{k=0}^{N}\left|\zeta\left(\sigma_{1}+i t+i k h+i \tau, \alpha\right)\right|\right) \mathrm{d} \tau\right) .
\end{aligned}
$$

Поскольку $1 / 2<\sigma_{1}<1$, то, принимая во внимание оценку (3.4), находим

$$
\begin{aligned}
& \frac{1}{N} \sum_{k=0}^{N}\left|\zeta\left(\sigma_{1}+i t+i k h+i \tau, \alpha\right)\right|=O\left(\frac{1}{N} \sum_{k=0}^{N}\left|\zeta\left(\sigma_{1}+i t+i k h+i \tau, \alpha\right)\right|^{2}\right)^{1 / 2} \\
& \quad=O(1+|\tau|+|t|)
\end{aligned}
$$

Кроме того, хорошо известные свойства гамма-функции и лемма 3 показывают, что при $N \rightarrow \infty$

$$
\frac{1}{N} \sum_{k=0}^{N}\left|R_{n}(\sigma+i t+i k h, \alpha)\right|=o(1)
$$

Теперь из оценок (3.7)-(3.10) получаем, что при $N \rightarrow \infty$

$$
\begin{aligned}
& \frac{1}{N+1} \sum_{k=0}^{N} \sup _{s \in K}\left|\zeta(s+i k h, \alpha)-\zeta_{n}(s+i k h, \alpha)\right| \\
& \quad=O\left(\sup _{s \in L} \int_{-\infty}^{\infty}\left|l_{n}\left(\sigma_{1}-\sigma+i \tau, \alpha\right)\right|(1+|t|+|\tau|) \mathrm{d} \tau\right)+o(1) .
\end{aligned}
$$

Можем считать, что $\sigma_{1}<\min _{\sigma+i t \in L} \sigma$. Поскольку $t$ ограничено при $s \in L$ и $\sigma_{1}-\sigma<0$, отсюда заключаем, что

$$
\lim _{n \rightarrow \infty} \limsup _{N \rightarrow \infty} \frac{1}{N+1} \sum_{k=0}^{\infty} \sup _{s \in K}\left|\zeta(s+i k h, \alpha)-\zeta_{n}(s+i k h, \alpha)\right|=0 .
$$

Это равенство и определение метрики $\rho$ доказывают равенство (3.1). Лемма 4 доказана. 
Приближение в среднем функции $\underline{\zeta}(s, \underline{\alpha}, \underline{\omega})$ функцией $\underline{\zeta}_{n}(s, \underline{\alpha}, \underline{\omega})$ (аналог леммы 4) является более сложным. Для этого мы привлечем некоторые факты эргодической теории.

Пусть $a_{\tau, \alpha}=\left((m+\alpha)^{-i \tau}: m \in \mathbb{N}_{0}\right), \tau \in \mathbb{R}, 0<\alpha<1$. Определим преобразование $\varphi_{\tau, \alpha}: \Omega \rightarrow \Omega$ формулой $\varphi_{\tau, \alpha}(\omega)=a_{\tau, \alpha} \omega, \omega \in \Omega$. Тогда $\left\{\varphi_{\tau, \alpha}: \tau \in \mathbb{R}\right\}-$ однопараметрическая группа измеримых сохраняющих меру преобразований тора $\Omega$. Множество $A \in \mathscr{B}(\Omega)$ называется инвариантным относительно группы $\varphi_{\tau, \alpha}$, если множества $A$ и $\varphi_{\tau, \alpha}(A)$ отличаются друг от друга самое большее на множество нулевой меры Хаара $m$ на $(\Omega, \mathscr{B}(\Omega))$. Группа $\left\{\varphi_{\tau, \alpha}: \tau \in \mathbb{R}\right\}$ называется эргодической, если $\sigma$-поле инвариантных множеств состоит только из множеств $A$, имеющих меру $m(A)=1$ или $m(A)=0$. Пусть

$$
L(\alpha)=\left\{\log (m+\alpha): m \in \mathbb{N}_{0}\right\} .
$$

Из [8; лемма 10] с $r=1$ вытекает следующее утверждение.

Лемма 5. Предположим, что множество $L(\alpha)$ линейно независимо над $\mathbb{Q}$. Тогда группа $\left\{\varphi_{\tau, \alpha}: \tau \in \mathbb{R}\right\}$ эргодична.

Последняя лемма позволяет оценить среднее квадратическое функции $\zeta(\sigma+$ it, $\alpha, \omega)$.

ЛЕмма 6. Предположим, что множество $L(\alpha)$ линейно независимо над $\mathbb{Q}$. Тогда при фиксированном $\sigma, 1 / 2<\sigma<1$, для почти всех $\omega \in \Omega$ имеет место оценка

$$
\sum_{k=0}^{N}|\zeta(\sigma+i t+i k h, \alpha, \omega)|^{2}=O(N(1+|t|)) .
$$

ДокАЗАТЕЛЬство. В [4; лемма 5.2.10] было установлено, что при трансцендентном $\alpha$ и $1 / 2<\sigma<1$ для почти всех $\omega \in \Omega$ имеет место оценка

$$
\int_{0}^{T}|\zeta(\sigma+i t, \alpha, \omega)|^{2} \mathrm{~d} t=O(T) .
$$

При этом трансцендентность числа $\alpha$ использовалась только для доказательства эргодичности группы $\left\{\varphi_{\tau, \alpha}: \tau \in \mathbb{R}\right\}$. Следовательно, в силу леммы 5 оценка (3.11) остается справедливой при линейной независимости множества $L(\alpha)$. Отсюда и из леммы 3 вытекает утверждение леммы.

ЛЕмма 7. Предположим, что множество $L(\alpha)$ линейно независимо над $\mathbb{Q}$. Тогда для почти всех $\omega \in \Omega$ имеет место равенство

$$
\lim _{n \rightarrow \infty} \limsup _{N \rightarrow \infty} \frac{1}{N+1} \sum_{k=0}^{\infty} \rho\left(\zeta(s+i k h, \alpha, \omega), \zeta_{n}(s+i k h, \alpha, \omega)\right)=0 .
$$

ДокАЗАТЕльство. Используя лемму 6, повторяем доказательство равенства (3.1).

ЛЕмма 8. Предположим, что множество $L\left(\alpha_{1}, \ldots, \alpha_{r}\right)$ линейно независимо над $\mathbb{Q}$. Тогда при любом $h>0$ для почти всех $\underline{\omega} \in \underline{\Omega}$ имеет место равенство

$$
\lim _{n \rightarrow \infty} \limsup _{N \rightarrow \infty} \frac{1}{N+1} \sum_{k=0}^{\infty} \underline{\rho}\left(\underline{\zeta}(s+i k h, \underline{\alpha}, \underline{\omega}), \underline{\zeta}_{n}(s+i k h, \underline{\alpha}, \underline{\omega})\right)=0 .
$$


ДокАЗАТЕльство. Как и в доказательстве леммы 4, имеем

$$
\begin{aligned}
& \frac{1}{N+1} \sum_{k=0}^{N} \underline{\rho}\left(\underline{\zeta}(s+i k h, \underline{\alpha}, \underline{\omega}), \underline{\zeta}_{n}(s+i k h, \underline{\alpha}, \underline{\omega})\right) \\
& \quad \leqslant \sum_{j=1}^{r} \frac{1}{N+1} \sum_{k=0}^{N} \rho\left(\zeta\left(s+i k h, \alpha_{j}, \omega_{j}\right), \zeta_{n}\left(s+i k h, \alpha_{j}, \omega_{j}\right)\right) .
\end{aligned}
$$

Из линейной независимости множества $L\left(\alpha_{1}, \ldots, \alpha_{r}\right)$ вытекает линейная независимость множеств $L\left(\alpha_{1}\right), \ldots, L\left(\alpha_{r}\right)$. Поэтому из леммы 7 следует, что для почти всех $\omega_{j} \in \Omega_{j}$

$$
\lim _{n \rightarrow \infty} \limsup _{N \rightarrow \infty} \frac{1}{N+1} \sum_{k=0}^{N} \rho\left(\zeta\left(s+i k h, \alpha_{j}, \omega_{j}\right), \zeta_{n}\left(s+i k h, \alpha_{j}, \omega_{j}\right)\right)=0
$$

при $j=1, \ldots, r$. Пусть равенство (3.13) выполняется для множества $\Omega_{0 j} \subset \Omega_{j}$, $j=1, \ldots, r$. Тогда $m_{j H}\left(\Omega_{0 j}\right)=1, j=1, \ldots, r$. Пусть $\underline{\Omega}_{0}=\Omega_{01} \times \cdots \times \Omega_{0 r}$. Так как мера $m_{H}$ является произведением мер $m_{j H}, j=1, \ldots, r$, отсюда получаем

$$
m_{H}\left(\underline{\Omega}_{0}\right)=m_{1 H}\left(\Omega_{01}\right) \cdots m_{r H}\left(\Omega_{0 r}\right)=1 .
$$

Поэтому утверждение леммы является следствием неравенства (3.12) и равенства (3.13).

На $\left(H^{r}(D), \mathscr{B}\left(H^{r}(D)\right)\right)$ определим еще одну вероятностную меру:

$$
\widehat{P}_{N}(A) \stackrel{\text { def }}{=} \frac{1}{N+1} \#\left\{0 \leqslant k \leqslant N: \underline{\zeta}_{n}(s+i k h, \underline{\alpha}, \underline{\omega}) \in A\right\} .
$$

Отметим, что мера $\widehat{P}_{N}(A)$ имеет смысл для почти всех $\underline{\omega} \in \underline{\Omega}$.

ЛЕмма 9. Предположим, что множество $L\left(\alpha_{1}, \ldots, \alpha_{r} ; h\right)$ линейно независимо над $\mathbb{Q}$. Тогда на $\left(H^{r}(D), \mathscr{B}\left(H^{r}(D)\right)\right)$ существует вероятностная мера $P$ такая, что обе меры $P_{N}$ и $\widehat{P}_{N}$ при $N \rightarrow \infty$ слабо сходятся $\kappa P$.

ДокАЗАтЕльство. Пусть дискретная случайная величина $\theta_{N}$ определена на некотором вероятностном пространстве $(\widehat{\Omega}, \mathscr{A}, \mathbb{P})$ и имеет место закон распределения

$$
\mathbb{P}\left(\theta_{N}=k\right)=\frac{1}{N+1}, \quad k=0, \ldots, N .
$$

На этом вероятностном пространстве определим $H^{r}(D)$-значный случайный элемент $\underline{X}_{N, n}$ формулой

$$
\underline{X}_{N, n}=\underline{X}_{N, n}(s)=\left(X_{N, n, 1}(s), \ldots, X_{N, n, r}(s)\right)=\underline{\zeta}_{n}\left(s+i \theta_{N} h, \underline{\alpha}\right) .
$$

Тогда, обозначая через $\underline{X}_{n}=\underline{X}_{n}(s) H^{r}(D)$-значный случайный элемент с распределением $P_{n}$, где $P_{n}-$ предельная мера в лемме 2 , и через $\stackrel{\mathscr{D}}{\longrightarrow}-$ сходимость по распределению, утверждение леммы 2 для меры $P_{N, n}$ можем записать в виде

$$
\underline{X}_{N, n} \underset{N \rightarrow \infty}{\stackrel{\mathscr{D}}{\longrightarrow}} \underline{X}_{n} .
$$

Теперь докажем, что семейство вероятностных мер $\left\{P_{n}: n \in \mathbb{N}\right\}$ является плотным; это означает, что всякому $\epsilon>0$ соответствует компактное множество 
$K=K(\epsilon) \subset H^{r}(D)$ такое, что $P_{n}(K)>1-\epsilon$ для всех $n \in \mathbb{N}$. Пусть $K_{l}-$ компактные множества из определения метрики $\rho$, а $M_{j l}$ - положительные числа, $j=1, \ldots, r, l \in \mathbb{N}$. Тогда имеем

$$
\begin{aligned}
\mathbb{P}(\exists j & \left.: \sup _{s \in K_{l}}\left|X_{N, n, j}(s)\right|>M_{j l}\right) \leqslant \sum_{j=1}^{r} \mathbb{P}\left(\sup _{s \in K_{l}}\left|X_{N, n, j}(s)\right|>M_{j l}\right) \\
& =\sum_{j=1}^{r} \frac{1}{(N+1)} \#\left\{0 \leqslant k \leqslant N: \sup _{s \in K_{l}}\left|\zeta_{n}\left(s+i k h, \alpha_{j}\right)\right|>M_{j l}\right\} \\
& \leqslant \sum_{j=1}^{r} \frac{1}{(N+1) M_{j l}} \sum_{k=0}^{N} \sup _{s \in K_{l}}\left|\zeta_{n}\left(s+i k h, \alpha_{j}\right)\right| .
\end{aligned}
$$

Поскольку ряд для $\zeta_{n}\left(s, \alpha_{j}\right)$ сходится абсолютно при $\sigma>1 / 2$, то для всех $n \in \mathbb{N}$

$$
\lim _{T \rightarrow \infty} \frac{1}{T} \int_{0}^{T}\left|\zeta_{n}\left(\sigma+i t, \alpha_{j}\right)\right|^{2} \mathrm{~d} t=\sum_{m=0}^{\infty} \frac{v_{n}^{2}\left(m, \alpha_{j}\right)}{\left(m+\alpha_{j}\right)^{2 \sigma}}<\sum_{m=0}^{\infty} \frac{1}{\left(m+\alpha_{j}\right)^{2 \sigma}}<\infty .
$$

Отсюда для всех $n \in \mathbb{N}$ и $1 / 2<\sigma<1$

$$
\int_{0}^{T}\left|\zeta_{n}\left(\sigma+i t, \alpha_{j}\right)\right|^{2} \mathrm{~d} t=O(T) .
$$

Эта оценка и интегральная формула Коши влекут за собой для всех $n \in \mathbb{N}$ оценку

$$
\int_{0}^{T}\left|\zeta_{n}^{\prime}\left(\sigma+i t, \alpha_{j}\right)\right|^{2} \mathrm{~d} t=O(T)
$$

Применяя лемму 3 , отсюда получаем, что для всех $n \in \mathbb{N}$ и $1 / 2<\sigma<1$

$$
\sum_{k=0}^{N}\left|\zeta_{n}\left(\sigma+i k h, \alpha_{j}\right)\right|^{2}=O(N)
$$

Отсюда опять в силу интегральной формулы Коши находим, что существуют константы $C_{j l}, 0<C_{j l}<\infty$, такие, что для всех $n \in \mathbb{N}$

$$
\frac{1}{N+1} \sum_{k=0}^{N} \sup _{s \in K_{l}}\left|\zeta_{n}\left(s+i k h, \alpha_{j}\right)\right| \leqslant C_{j l} .
$$

Пусть $\epsilon$ - любое положительное число, а $M_{j l}=2^{l} \epsilon^{-1} r C_{j l}, j=1, \ldots, r, l \in \mathbb{N}$. Тогда из неравенств (3.15) и (3.16) следует, что для всех $n \in \mathbb{N}$ и $l \in \mathbb{N}$

$$
\mathbb{P}\left(\exists j: \sup _{s \in K_{l}}\left|X_{N, n, j}(s)\right|>M_{j l}\right) \leqslant \frac{\epsilon}{2^{l}} .
$$

Отсюда получаем, что для всех $n \in \mathbb{N}$

$$
\mathbb{P}\left(\sup _{s \in K_{l}}\left|X_{N, n, j}(s)\right| \leqslant M_{j l}, j=1, \ldots, r, l \in \mathbb{N}\right) \geqslant 1-\epsilon \sum_{l=1}^{\infty} \frac{1}{2^{l}}=1-\epsilon,
$$

и в силу (3.14) для всех $n \in \mathbb{N}$

$$
\mathbb{P}\left(\sup _{s \in K_{l}}\left|X_{n, j}(s)\right| \leqslant M_{j l}, j=1, \ldots, r, l \in \mathbb{N}\right) \geqslant 1-\epsilon .
$$


Пусть

$$
H_{\epsilon}^{r}=\left\{\left(g_{1}, \ldots, g_{r}\right) \in H^{r}(D): \sup _{s \in K_{j}}\left|g_{j}(s)\right| \leqslant M_{j l}, j=1, \ldots, r, l \in \mathbb{N}\right\} .
$$

Тогда $H_{\epsilon}^{r}$ - компактное множество пространства $H^{r}(D)$ и ввиду $(3.17)$ для всех $n \in \mathbb{N}$

$$
\mathbb{P}\left(\underline{X}_{n} \in H_{\epsilon}^{r}\right) \geqslant 1-\epsilon,
$$

или, по определению, $\underline{X}_{n}$ для всех $n \in \mathbb{N}$

$$
P_{n}\left(H_{\epsilon}^{r}\right) \geqslant 1-\epsilon,
$$

т.е. семейство $\left\{P_{n}: n \in \mathbb{N}\right\}$ плотно.

Напоминаем, что семейство вероятностных мер относительно компактно, если каждая его последовательность содержит слабо сходящуюся к некоторой мере подпоследовательность. Прямая теорема Прохорова (см. [16; теорема 6.1]) утверждает, что всякая плотная последовательность вероятностных мер относительно компактна. Следовательно, имеем, что последовательность $\left\{P_{n}: n \in \mathbb{N}\right\}$ относительно компактна. Поэтому существует последовательность $\left\{P_{n_{k}}\right\} \subset\left\{P_{n}\right\}$ такая, что $P_{n_{k}}$ при $k \rightarrow \infty$ слабо сходится к некоторой мере $P$ на $\left(H^{r}(D), \mathscr{B}\left(H^{r}(D)\right)\right)$. Этот факт, используя определение случайного элемента $X_{n}$, можем записать соотношением

$$
\underline{X}_{n_{k}} \underset{k \rightarrow \infty}{\stackrel{\mathscr{D}}{\longrightarrow}} P
$$

Пусть еще

$$
\underline{X}_{N}=\underline{X}_{N}(s)=\underline{\zeta}\left(s+i \theta_{N} h, \underline{\alpha}\right) .
$$

Тогда, воспользовавшись леммой 4 , находим, что для всякого $\epsilon>0$

$$
\begin{aligned}
& \lim _{n \rightarrow \infty} \limsup _{N \rightarrow \infty} \mathbb{P}\left(\underline{\rho}\left(\underline{X}_{N}, \underline{X}_{N, n}\right) \geqslant \epsilon\right) \\
& \quad=\lim _{n \rightarrow \infty} \limsup _{N \rightarrow \infty} \frac{1}{N+1} \#\left\{0 \leqslant k \leqslant N: \underline{\rho}\left(\underline{\zeta}(s+i k h, \underline{\alpha}), \underline{\zeta}_{n}(s+i k h, \underline{\alpha})\right) \geqslant \epsilon\right\} \\
& \quad \leqslant \lim _{n \rightarrow \infty} \limsup _{N \rightarrow \infty} \frac{1}{N+1} \sum_{k=0}^{N} \underline{\rho}\left(\underline{\zeta}(s+i k h, \underline{\alpha}), \underline{\zeta}_{n}(s+i k h, \underline{\alpha})\right)=0 .
\end{aligned}
$$

Соотношения (3.14), (3.18) и (3.19) показывают, что случайные элементы $\underline{X}_{N}$, $\underline{X}_{N, n}$ и $\underline{X}_{n_{k}}$ Удовлетворяют условиям теоремы 4.2 из [16]. Поэтому получаем

$$
\underline{X}_{N} \underset{N \rightarrow \infty}{\stackrel{\mathscr{D}}{\longrightarrow}} P
$$

что эквивалентно слабой сходимости $P_{N}$ к $P$ при $N \rightarrow \infty$.

Соотношение (3.20) показывает, что мера $P$ не зависит от выбора последовательности $\left\{P_{n_{k}}\right\}$. Последнее означает, что соотношение (3.18) может быть заменено на

$$
\underline{X}_{n} \underset{n \rightarrow \infty}{\stackrel{\mathscr{D}}{\longrightarrow}} P
$$

Остается показать, что мера $\widehat{P}_{N}$ при $N \rightarrow \infty$ также слабо сходится к $P$. На вероятностном пространстве $(\widehat{\Omega}, \mathscr{A}, \mathbb{P})$ определим $H^{r}(D)$-значные случайные элементы

$$
\underline{\widehat{X}}_{N, n}=\underline{\widehat{X}}_{N, n}(s)=\underline{\zeta}_{n}\left(s+i \theta_{N} h, \underline{\alpha}, \underline{\omega}\right), \quad \underline{\widehat{X}}_{N}=\underline{\widehat{X}}_{N}(s)=\underline{\zeta}\left(s+i \theta_{N} h, \underline{\alpha}, \underline{\omega}\right) .
$$


Тогда, повторяя для $\widehat{\widehat{X}}_{N}$ и $\underline{\widehat{X}}_{N, n}$ рассуждения, аналогичные примененным для случайных элементов $\underline{X}_{N}$ и $\underline{X}_{N, n}$, и применяя соотношение $(3.21)$ и лемму 8 , убеждаемся, что мера $\widehat{P}_{N}$ при $N \rightarrow \infty$ также слабо сходится к мере $P$.

Лемма 9 доказана.

Для доказательства теоремы 4 нам еще нужна эргодичность одного преобразования группы $\underline{\Omega}$. Пусть

$$
a_{\underline{\alpha}, h}=\left(\left(\left(m+\alpha_{1}\right)^{-i h}: m \in \mathbb{N}_{0}\right), \ldots,\left(\left(m+\alpha_{r}\right)^{-i h}: m \in \mathbb{N}_{0}\right)\right) .
$$

Определим преобразование группы $\underline{\Omega}$ :

$$
\varphi_{\underline{\alpha}, h}(\underline{\omega})=a_{\underline{\alpha}}, \underline{\omega}, \quad \underline{\omega} \in \underline{\Omega} .
$$

Тогда $\varphi_{\underline{\alpha}, h}$ является измеримым сохраняющим меру преобразованием на вероятностном пространстве $\left(\underline{\Omega}, \mathscr{B}(\underline{\Omega}), m_{H}\right)$. Множество $A \in \mathscr{B}(\underline{\Omega})$ называется инвариантным множеством преобразования $\varphi_{\underline{\alpha}, h}$, если множества $A$ и $\varphi_{\underline{\alpha}, h}(A)$ отличаются друг от друга самое большее на множество нулевой меры $m_{H}$.

ЛЕмма 10. Преобразование $\varphi_{\underline{\alpha}, h}$ эргодично, т.е. $\sigma$-поле его инвариантных множеств состоит только из множеств $А$ с $m_{H}(A)=1$ или $m_{H}(A)=0$.

ДокАЗАТЕЛьство. Будем применять метод преобразований Фурье. При доказательстве леммы 1 мы видели, что характеры $\chi$ группы $\underline{\Omega}$ имеют вид

$$
\chi(\underline{\omega})=\prod_{j=1}^{r} \prod_{m=0}^{\infty} \omega_{j}^{k_{j m}}(m),
$$

где только конечное число целых чисел $k_{j m}$ отличны от нуля. Сначала пусть $\chi$ - нетривиальный характер. Тогда в силу неравенства (2.3)

$$
\chi\left(a_{\underline{\alpha}, h}\right)=\exp \left\{-i h \sum_{j=1}^{r} \sum_{m=0}^{\infty} k_{j m} \log \left(m+\alpha_{j}\right)\right\} \neq 1
$$

при $\underline{k} \neq \underline{0}$. Пусть $A$ - любое инвариантное множество преобразования $\varphi_{\underline{\alpha}}, h$, а $I_{A}$ - индикатор множества $A$. Тогда имеем, что для почти всех $\underline{\omega} \in \underline{\Omega}$

$$
I_{A}\left(a_{\underline{\alpha}}, \underline{\omega}\right)=I_{A}(\underline{\omega}) .
$$

Через $\widehat{f}$ будем обозначать преобразование Фурье функции $f$. Тогда из равенства (3.23) и инвариантности меры Хаара $m_{H}$ имеем

$$
\begin{aligned}
\widehat{I}_{A}(\chi) & =\int_{\underline{\Omega}} \chi(\underline{\omega}) I_{A}(\underline{\omega}) m_{H}(\mathrm{~d} \underline{\omega})=\int_{\underline{\Omega}} \chi\left(a_{\underline{\alpha}}, h \underline{\omega}\right) I_{A}\left(a_{\underline{\alpha}}, h \underline{\omega}\right) m_{H}(\mathrm{~d} \underline{\omega}) \\
& =\chi\left(a_{\underline{\alpha}, h}\right) \widehat{I}_{A}(\chi) .
\end{aligned}
$$

Отсюда и из неравенства (3.23) находим, что

$$
\widehat{I}_{A}(\chi)=0
$$

для каждого нетривиального характера $\chi$ группы $\underline{\Omega}$. 
Теперь пусть $\chi_{0}-$ тривиальный характер группы $\underline{\Omega}$, т.е. $\chi_{0}(\underline{\omega}) \equiv 1$. Пусть $\widehat{I}_{A}\left(\chi_{0}\right)=u$. Тогда из равенств

$$
\int_{\Omega} \chi(\underline{\omega}) m_{H}(\mathrm{~d} \underline{\omega})= \begin{cases}1, & \text { если } \chi=\chi_{0}, \\ 0, & \text { если } \chi \neq \chi_{0},\end{cases}
$$

и (3.24) получаем, что для любого характера $\chi$ группы $\underline{\Omega}$

$$
\widehat{I}_{A}(\chi)=u \int_{\underline{\Omega}} \chi(\underline{\omega}) m_{H}(\mathrm{~d} \underline{\omega})=u \widehat{1}(\chi)=\widehat{u}(\chi) .
$$

Поскольку функция $I_{A}(\underline{\omega})$ однозначно определяется ее преобразованием Фурье $\widehat{I}_{A}(\chi)$, то отсюда получаем, что для почти всех $\underline{\omega} \in \underline{\Omega}$ имеет место равенство $I_{A}(\underline{\omega})=u$. Отсюда $u=0$ или $u=1$. Поэтому $I_{A}(\underline{\omega})=0$ для почти всех $\underline{\omega} \in \underline{\Omega}$ или же $I_{A}(\underline{\omega})=1$. Следовательно, $m_{H}(A)=0$ или $m_{H}(A)=1$. Лемма доказана.

Сейчас мы готовы доказать теорему 4.

ДокАЗАТЕЛЬСтво тЕоремы 4. Из леммы 9 вытекает, что достаточно показать, что предельная мера $P$ в лемме 9 совпадает с $P_{\zeta}$.

Пусть $A$ - любое множество непрерывности меры $P$, т.е. $P(\partial A)=0$, где $\partial A-$ граница множества $A$. Тогда из леммы 9 и эквивалента слабой сходимости вероятностных мер в терминах множеств непрерывности (см. [16; теорема 2.1]) имеем

$$
\lim _{N \rightarrow \infty} \frac{1}{N+1} \#\{0 \leqslant k \leqslant N: \underline{\zeta}(s+i k h, \underline{\alpha}, \underline{\omega}) \in A\}=P(A) .
$$

На вероятностном пространстве $\left(\underline{\Omega}, \mathscr{B}(\underline{\Omega}), m_{H}\right)$ определим случайную величину $\xi$ формулой

$$
\xi(\underline{\omega})= \begin{cases}1, & \text { если } \underline{\zeta}(s, \underline{\alpha}, \underline{\omega}) \in A, \\ 0 & \text { в противном случае. }\end{cases}
$$

Тогда, обозначая через $\mathbb{E} \xi$ математическое ожидание случайной величины $\xi$, имеем

$$
\mathbb{E} \xi=\int_{\underline{\Omega}} \xi \mathrm{d} m_{H}=m_{H}(\underline{\omega} \in \underline{\Omega}: \underline{\zeta}(s, \underline{\alpha}, \underline{\omega}) \in A)=P_{\underline{\zeta}}(A) .
$$

Поскольку преобразование $\varphi_{\underline{\alpha}, h}$ эргодично, то из классической теоремы Биркгофа-Хинчина следует, что для почти всех $\underline{\omega} \in \underline{\Omega}$

$$
\lim _{N \rightarrow \infty} \frac{1}{N+1} \sum_{k=0}^{N} \xi\left(\varphi_{\underline{\alpha}, h}^{k}(\underline{\omega})\right)=\mathbb{E} \xi .
$$

С другой стороны, определения случайной величины $\xi$ и преобразования $\varphi_{\underline{\alpha}, h}$ показывают, что

$$
\frac{1}{N+1} \sum_{k=0}^{N} \xi\left(\varphi_{\underline{\alpha}, h}^{k}(\underline{\omega})\right)=\frac{1}{N+1} \#\{0 \leqslant k \leqslant N: \underline{\zeta}(s+i k h, \underline{\alpha}, \underline{\omega}) \in A\} .
$$

Поэтому, учитывая (3.26) и (3.27), получаем, что для почти всех $\underline{\omega} \in \underline{\Omega}$

$$
\lim _{N \rightarrow \infty} \frac{1}{N+1} \#\{0 \leqslant k \leqslant N: \underline{\zeta}(s+i k h, \underline{\alpha}, \underline{\omega}) \in A\}=P_{\underline{\zeta}}(A) .
$$


Отсюда и из равенства (3.25) заключаем, что $P(A)=P_{\underline{\zeta}}(A)$ для всех множеств непрерывности $A$ меры $P$. Поскольку множества непрерывности образуют определяющий класс (см. [16]), то $P(A)=P_{\underline{\zeta}}(A)$ для всех $A \in \mathscr{B}(H(D))$, т.е. мера $P$ совпадает с $P_{\underline{\zeta}}$.

Теорема 4 доказана.

\section{§ 4. Доказательство теоремы универсальности}

Основным ингредиентом доказательства теоремы 4 является теорема 6, однако еще нужен явный вид носителя предельной меры $P_{\underline{\zeta}}$. Напоминаем, что носителем меры $P_{\underline{\zeta}}$ называется минимальное замкнутое множество $S_{\underline{\zeta}} \subset H^{r}(D)$ такое, что $P_{\underline{\zeta}}\left(S_{\underline{\zeta}}\right)^{-}=1$. Множество $S_{\underline{\zeta}}$ состоит из всех элементов $\underline{g} \in H^{r}(D)$ таких, что для всякой открытой окрестности $G$ элемента $\underline{g}$ выполняется неравенство $P_{\underline{\zeta}}(G)>0$.

ЛЕмма 11. Предположим, что множество $L\left(\alpha_{1}, \ldots, \alpha_{r} ; h\right)$ линейно независимо над $\mathbb{Q}$. Тогда носителем меры $P_{\underline{\zeta}}$ является все пространство $H^{r}(D)$.

ДокАЗАтЕльство. При доказательстве теоремы 3 в предположении, что множество $L\left(\alpha_{1}, \ldots, \alpha_{r}\right)$ линейно независимо над $\mathbb{Q}$, в [8] было получено, что носителем меры $P_{\underline{\zeta}}$ является все пространство $H^{r}(D)$. Так как из линейной независимости множества $L\left(\alpha_{1}, \ldots, \alpha_{r} ; h\right)$ вытекает линейная независимость множества $L\left(\alpha_{1}, \ldots, \alpha_{r}\right)$, то отсюда получаем утверждение леммы.

Нам еще будет нужна теорема Мергеляна о приближении аналитических функций многочленами. Мы сформулируем ее в виде отдельной леммы в удобном для нас виде.

ЛЕмма 12. Предположим, что $K$ - компактное подмножество комплексной плоскости, обладающее связным дополнением, а функиия $f(s)$ непрерывна в $K$ и аналитична внутри $K$. Тогда для всякого $\epsilon>0$ существует многочлен $p(s)$ такой, что

$$
\sup _{s \in K}|f(s)-p(s)|<\epsilon .
$$

Доказательство леммы дано в [18], оно также содержится в [19].

ДОКАЗАТЕЛЬСТВО ТЕОРЕМЫ 5. В силу леммы 12 найдутся многочлены $p_{1}(s), \ldots, p_{r}(s)$ такие, что

$$
\sup _{1 \leqslant j \leqslant r} \sup _{s \in K_{j}}\left|f_{j}(s)-p_{j}(s)\right|<\frac{\epsilon}{2} .
$$

Определим множество

$$
G=\left\{\left(g_{1}, \ldots, g_{r}\right) \in H^{r}(D): \sup _{1 \leqslant j \leqslant r} \sup _{s \in K_{j}}\left|g_{j}(s)-p_{j}(s)\right|<\frac{\epsilon}{2}\right\} .
$$

Тогда $G$ - открытое множество пространства $H^{r}(D)$. Кроме того, в силу леммы 11 получаем, что оно является окрестностью элемента $\left(p_{1}, \ldots, p_{r}\right)$, принадлежащего носителю меры $P_{\underline{\zeta}}$. Поэтому

$$
P_{\underline{\zeta}}(G)>0 .
$$


Воспользовавшись эквивалентом слабой сходимости вероятностных мер в терминах открытых множеств (см. [16; теорема 2.1]), из теоремы 4 и определения меры $P_{N}$ выводим, что

$$
\liminf _{N \rightarrow \infty} \frac{1}{N+1} \#\{0 \leqslant k \leqslant N: \underline{\zeta}(s+i k h, \underline{\alpha}) \in G\} \geqslant P_{\underline{\zeta}}(G) \text {. }
$$

Отсюда, из неравенства (4.2) и определения множества $G$ следует, что

$$
\liminf _{N \rightarrow \infty} \frac{1}{N+1} \#\left\{0 \leqslant k \leqslant N: \sup _{1 \leqslant j \leqslant r} \sup _{s \in K_{j}}\left|\zeta\left(s+i k h, \alpha_{j}\right)-p_{j}(s)\right|<\frac{\epsilon}{2}\right\}>0 .
$$

Остается заменить в этом неравенстве многочлены $p_{j}(s)$ функциями $f_{j}(s), j=$ $1, \ldots, r$. Пусть $k \in \mathbb{N}_{0}$ удовлетворяет неравенству

$$
\sup _{1 \leqslant j \leqslant r} \sup _{s \in K_{j}}\left|\zeta\left(s+i k h, \alpha_{j}\right)-p_{j}(s)\right|<\frac{\epsilon}{2} .
$$

Тогда для таких $k$, учитывая неравенство (4.1), получаем

$$
\sup _{1 \leqslant j \leqslant r} \sup _{s \in K_{j}}\left|\zeta\left(s+i k h, \alpha_{j}\right)-f_{j}(s)\right|<\epsilon .
$$

Следовательно,

$$
\begin{aligned}
\{0 \leqslant & \left.k \leqslant N: \sup _{1 \leqslant j \leqslant r} \sup _{s \in K_{j}}\left|\zeta\left(s+i k h, \alpha_{j}\right)-p_{j}(s)\right|<\frac{\epsilon}{2}\right\} \\
& \subset\left\{0 \leqslant k \leqslant N: \sup _{1 \leqslant j \leqslant r} \sup _{s \in K_{j}}\left|\zeta\left(s+i k h, \alpha_{j}\right)-f_{j}(s)\right|<\epsilon\right\} .
\end{aligned}
$$

Отсюда и из неравенства (4.3) получаем

$$
\liminf _{N \rightarrow \infty} \frac{1}{N+1} \#\left\{0 \leqslant k \leqslant N: \sup _{1 \leqslant j \leqslant r} \sup _{s \in K_{j}}\left|\zeta\left(s+i k h, \alpha_{j}\right)-f_{j}(s)\right|<\epsilon\right\}>0 .
$$

Теорема 5 доказана.

\section{§5. Доказательство теоремы 6}

Теорема 6 является следствием теоремы 5 и классической теоремы Руше, которую для удобства сформулируем в виде следующей леммы.

ЛЕмма 13. Пусть функции $f(s)$ и $g(s)$ аналитичны внутри и на контуре $\Gamma$, а на Г удовлетворяют условиям $f(s) \neq 0 u|g(s)|<|f(s)|$. Тогда внутри Г функиии $f(s)$ и $f(s)+g(s)$ имеют одинаковое число нулей.

Доказательство леммы дано, например, в [20].

ДОКАЗАТЕЛЬСТВо ТЕОРЕмЫ 6. Пусть

$$
r=\frac{\sigma_{2}-\sigma_{1}}{2}, \quad C=\sum_{j=1}^{r}\left|c_{j}\right| .
$$

Не ограничивая общности, можем считать, что $c_{1} \neq 0$. Пусть

$$
f_{1}(s)=c_{1}^{-1}(s-\widehat{\sigma}), \quad f_{2}(s)=\cdots=f_{r}(s)=\epsilon>0,
$$


где число $\epsilon$ удовлетворяет неравенству

$$
C \epsilon<\frac{1}{20} \min _{|s-\widehat{\sigma}|=r}|s-\widehat{\sigma}|=\frac{r}{20} .
$$

Предположим, что $k \in \mathbb{N}_{0}$ удовлетворяет неравенству

$$
\sup _{1 \leqslant j \leqslant r} \sup _{|s-\widehat{\sigma}| \leqslant r}\left|\zeta\left(s+i k h, \alpha_{j}\right)-f_{j}(s)\right|<\epsilon .
$$

Отсюда для таких $k$ получаем

$$
\sup _{|s-\widehat{\sigma}| \leqslant r}\left|\sum_{j=1}^{r} c_{j} \zeta\left(s+i k h, \alpha_{j}\right)-\sum_{j=1}^{r} c_{j} f_{j}(s)\right|<C \epsilon .
$$

Поэтому в силу определения функций $f_{1}(s), \ldots, f_{r}(s)$

$$
\sup _{|s-\widehat{\sigma}|=r}\left|\sum_{j=1}^{r} c_{j} \zeta\left(s+i k h, \alpha_{j}\right)-(s-\widehat{\sigma})\right|<2 C \epsilon .
$$

Неравенства (5.1) и (5.2) показывают, что функции

$$
\sum_{j=1}^{r} c_{j} \zeta\left(s+i k h, \alpha_{j}\right)-(s-\widehat{\sigma})
$$

и $s-\widehat{\sigma}$ в круге $|s-\widehat{\sigma}| \leqslant r$ удовлетворяют условиям леммы 13 . Так как функция $s-\widehat{\sigma}$ в круге имеет точно один нуль $s=\widehat{\sigma}$, то по лемме 13 и функция

$$
\sum_{j=1}^{r} c_{j} \zeta\left(s+i k h, \alpha_{j}\right)
$$

также имеет один нуль в этом круге. Однако по теореме 5 найдется константа $c=c\left(\sigma_{1}, \sigma_{2}, \underline{\alpha}, \underline{c}, h\right)>0$ такая, что при достаточно большом $N$

$$
\frac{1}{N+1} \#\left\{0 \leqslant k \leqslant N: \sup _{1 \leqslant j \leqslant r} \sup _{|s-\widehat{\sigma}| \leqslant r}\left|\zeta\left(s+i k h, \alpha_{j}\right)-f_{j}(s)\right|<\epsilon\right\}>c
$$

что и доказывает теорему 6.

\section{Список литературы}

[1] С. М. Воронин, Аналитические свойства производящих функиий Дирихле арифметических объектов, Дисс. ... докт. физ.-матем. наук, МИАН, М., 1977, 76 с.

[2] S. M. Gonek, Analytic properties of zeta and L-functions, Ph. D. thesis, University of Michigan, Michigan, USA, 1979, 175 pp.

[3] B. Bagchi, The statistical behaviour and universality properties of the Riemann zeta-function and other allied Dirichlet series, Ph. D. thesis, Indian Statistical Institute, Calcutta, 1981.

[4] A. Laurinčikas, R. Garunkštis, The Lerch zeta-function, Kluwer Academic Publishers, Dordrecht, 2002, viii+189 pp.

[5] J. Sander, J. Steuding, "Joint universality for sums and products of Dirichlet L-functions", Analysis (Munich), 26:3 (2006), 295-312. 
[6] A. Laurinčikas, R. Macaitienè, "The discrete universality of the periodic Hurwitz zeta function", Integral Transforms Spec. Funct., 20:9-10 (2009), 673-686.

[7] T. Nakamura, "The existence and the non-existence of joint $t$-universality for Lerch zeta-functions", J. Number Theory, 125:2 (2007), 424-441.

[8] A. Laurinčikas, "The joint universality of Hurwitz zeta-functions", Šiauliai Math. Semin., 3:11 (2008), 169-187.

[9] A. Laurinčikas, "On the universality of the Hurwitz zeta-function", Int. J. Number Theory, 9:1 (2013), 155-165.

[10] A. Laurinčikas, J. Rašytè, "Generalizations of a discrete universality theorem for Hurwitz zeta-functions", Lith. Math. J., 52:2 (2012), 172-180.

[11] A. Laurinčikas, "Joint universality of Hurwitz zeta-functions", Bull. Aust. Math. Soc., $\mathbf{8 6 : 2}$ (2012), 232-243.

[12] K. M. Bitar, N. N. Khuri, H. C. Ren, "Paths integrals and Voronin's theorem on the universality of the Riemann zeta function", Ann. Physics, 211:1 (1991), 172-196.

[13] С. М. Воронин, "Теорема об “универсальности” дзета-функции Римана", Изв. АН СССР. Сер. матем., 39:3 (1975), 475-486; англ. пер.: S. M. Voronin, "Theorem on the "universality" of the Riemann zeta-function", USSR-Izv., 9:3 (1975), 443-453.

[14] Ю.В. Нестеренко, "Модулярные функции и вопросы трансцендентности", Maтем. сб., 187:9 (1996), 65-96; англ. пер.: Yu. V. Nesterenko, "Modular functions and transcendence questions", Sb. Math., 187:9 (1996), 1319-1348.

[15] H. Heyer, Probability measures on locally compact groups, Ergeb. Math. Grenzgeb., 94, Springer-Verlag, Berlin-New York, 1977, x+531 pp.

[16] P. Billingsley, Convergence of probability measures, John Wiley \& Sons, Inc., New York-London-Sydney, 1968, xii+253 pp.

[17] H. L. Montgomery, Topics in multiplicative number theory, Lecture Notes in Math., 227, Springer-Verlag, Berlin-New York, 1971, ix+178 pp.

[18] С.Н. Мергелян, "Равномерные приближения функций комплексного перменного", УМН, 7:2(48) (1952), 31-122; англ. пер.: S. N. Mergelyan, "Uniform approximations to functions of a complex variable", Amer. Math. Soc. Transl., 101 (1954), $99 \mathrm{pp}$.

[19] Ж.Н. Уолш, Интерполячия и аппроксимация рациональными функциями в комплексной области, ИЛ, М., 1961, 508 с.; пер. с англ.: J. L. Walsh, Interpolation and approximation by rational functions in the complex domain, 3rd ed., Amer. Math. Soc. Colloq. Publ., XX, Amer. Math. Soc., Providence, R.I., 1960, x+398 pp.

[20] И.И. Привалов, Введение в теорию функиий комплексного переменного, 11-е изд., Наука, М., 1967, 444 с.

Антанас Лауринчикас

(Antanas Laurinčikas)

Вильнюсский университет, Литва

E-mail: antanas.laurincikas@mif.vu.lt
Поступила в редакцию 31.03.2014 и 27.06.2014 